Corrigendum

\title{
Corrigendum to "Design of the NIDA clinical trials network validation study of tobacco, alcohol, prescription medications, and substance use/misuse (TAPS) tool" [Contemporary Clinical Trials. 2016 Jul. 19; 50: 90-97]
}

\author{
Li-Tzy Wu $^{\text {a,b,c,d,* }}$, Jennifer McNeely ${ }^{\mathrm{e}}$, Geetha A. Subramaniam ${ }^{\mathrm{f}}$, Gaurav Sharma ${ }^{\mathrm{g}}$, \\ Paul VanVeldhuisen ${ }^{\mathrm{g}}$, Robert P. Schwartz ${ }^{\mathrm{h}}$ \\ a Department of Psychiatry and Behavioral Sciences, Duke University Medical Center, Durham, NC, USA \\ ${ }^{\mathrm{b}}$ Department of Medicine, Division of General Internal Medicine, Duke University Medical Center, Durham, NC, USA \\ c Duke Clinical Research Institute, Duke University Medical Center, Durham, NC, USA \\ ' Center for Child and Family Policy, Duke University, Durham, NC, USA \\ e Department of Population Health, New York University School of Medicine, New York, NY, USA \\ ${ }^{\mathrm{f}}$ National Institute on Drug Abuse, Bethesda, MD, USA \\ 'The EMMES Corporation, Rockville, MD, USA \\ ${ }^{\mathrm{h}}$ Friends Research Institute, Inc., Baltimore, MD, USA
}

In the article, we mistakenly stated that we drew select items from the World Health Organization's Composite International Diagnostic Interview 2.0 (WHO CIDI-2.0) substance abuse module to assess substance use disorders. However, we actually used select items from the WHO World Mental Health-CIDI [WMH-CIDI]: http://www.hcp.med. harvard.edu/wmhcidi/index.php). Additionally, the DSM-5 has only one set of criteria for all substance use disorders, including tobacco.
Because the WMH-CIDI does not contain all the criteria for DSM-5 Tobacco Use Disorder, we adapted the language from the WMHCIDI items from the drug section to assess tobacco use disorder. Readers of our article should note the mislabeling "modified CIDI2 " in Fig. 1 and on page 95. The correct description should read "modified WMH-CIDI."

The authors would like to apologise for any inconvenience caused.

\footnotetext{
DOI of original article: http://dx.doi.org/10.1016/j.cct.2016.07.013.

* Corresponding author at: Department of Psychiatry and Behavioral Sciences, Duke University Medical Center, Box 3903, Durham, NC, USA.

E-mail address: Litzy.wu@duke.edu (L.-T. Wu).
} 